383 FIRST-IN-MAN CLINICAL TRIAL OF INTRATUMORAL INJECTION OF CLOSTRIDIUM NOVYI-NT SPORES IN COMBINATION WITH PEMBROLIZUMAB IN PATIENTS WITH TREATMENT-REFRACTORY ADVANCED SOLID TUMORS

${ }^{1}$ Filip Janku*, ${ }^{2}$ Siqing Fu, ${ }^{2}$ Ravi Murthy, ${ }^{2}$ Daniel Karp, ${ }^{2}$ David Hong, ${ }^{2}$ Apostolia Tsimberidou, ${ }^{2}$ Maura Gillison, ${ }^{2}$ Abha Adat, ${ }^{2}$ Anjali Raina, ${ }^{2}$ Greg Call, ${ }^{3}$ Brent Kreider, ${ }^{3}$ David Tung, ${ }^{3}$ Mary Varterasian, ${ }^{4}$ Khashayarsha Khazaie. ${ }^{1}$ The University of Texas MD Anderson Cancer Center, Houston, TX, USA; ${ }^{2}$ MD Anderson Cancer Center, Houston, TX, USA; ${ }^{3}$ BioMed Valley Discoveries Inc., Kansas City, MO, USA; ${ }^{4}$ Mayo Clinic, Rochester, MN, USA

Background Intratumorally injected Clostridium novyi-NT (non-toxic), an attenuated strain of C. novyi that lacks production of the lethal alpha toxin, replicates within hypoxic tumor regions and elicits tumor-confined cell lysis. Early clini$\mathrm{cal}$ and translational data suggest that intratumoral injection of C. novyi-NT is feasible, demonstrates early signals of antitumor activity and induction of the host immune response, which supports additional studies in combination with immune checkpoint inhibitors.

Methods This first-in-human study (NCT03435952) enrolls patients with injectable, treatment-refractory solid tumors to receive a single intratumoral injection of C. novyi-NT across 4 dose cohorts $(3 \times 104$ to $100 \times 104$ spores, $3+3$ dose-escalation design) in combination with intravenous pembrolizumab $200 \mathrm{mg}$ every 3 weeks for up to 24 months to determine dose-limiting toxicities (DLTs), and the maximum tolerated dose (MTD).

Results As of August 24, 2020, 9 patients (breast cancer, $n=2$; colorectal cancer, $\mathrm{n}=1$; fibrous histiocytoma, $\mathrm{n}=1$; anal cancer, $\mathrm{n}=1$; chondrosarcoma, $\mathrm{n}=1$; appendiceal cancer, $\mathrm{n}=1$; tongue squamous cell cancer, $n=1$; nasopharyngeal cancer, $n=1$ ) were treated. There were no DLTs to date. Signs and symptoms of C. novyi-NT germination (infection) including fever, injection site pain, erythema, swelling, tenderness, and in some cases, ulceration, spontaneous drainage, tissue sloughing, bleeding, and malodor were observed in 5 patients. Partial responses were noted in 2 of 9 patients (tongue squamous cell cancer, nasopharyngeal cancer).

Conclusions Single intratumoral injection of C. novyi-NT in combination with pembrolizumab has been demonstrating manageable toxicity profile and encouraging signals of anticancer activity. The enrolment continues.

Trial Registration NCT03435952

Ethics Approval The study was approved by MD Anderson IRB

http://dx.doi.org/10.1136/jitc-2020-SITC2020.0383

\section{A PHASE 1 STUDY TO EVALUATE THE SAFETY, PK, AND ANTITUMOR ACTIVITY OF AK117, AN ANTI-CD47 MONOCLONAL ANTIBODY, IN SUBJECTS WITH RELAPSED/REFRACTORY ADVANCED OR METASTATIC SOLID TUMORS OR LYMPHOMAS}

\footnotetext{
${ }^{1}$ Amy Prawira*, ${ }^{2}$ Jermaine Coward, ${ }^{3}$ Anna Mislang, ${ }^{4}$ Adnan Nagrial, ${ }^{5}$ Hui Gan, ${ }^{6}$ Xiaoping Jin, ${ }^{6}$ Baiyong Li, ${ }^{6}$ Zhongmin Maxwell Wang, ${ }^{6}$ Kon Yew Kwek, ${ }^{6}$ Dennis Xia, ${ }^{6}$ Yu Xia. ${ }^{1}$ St Vincent's Hospital, Sydney, Australia; ${ }^{2}$ Icon Cancer Centre, Brisbane, Australia; ${ }^{3}$ Adelaide Cancer Centre, Kurralta Park, Australia; ${ }^{4}$ Blacktown Hospital, Blacktown, Australia; ${ }^{5}$ Austin Health, Melbourne, Victoria, Australia; ${ }^{6}$ Akeso Biopharma Inc, Zhongshan city, China
}

Background AK117 is a novel humanized IgG4 monoclonal antibody (mAb) targeting CD47, a macrophage immune checkpoint and 'don't eat me' signal that allows tumor cells to evade immune destruction by phagocytic cells. Most human cancers express CD47 to varying degrees, making CD47 a universal target. The efficacy of anti-CD47 therapy has been studied, as monotherapy and in combination with other antineoplastic agents, in various malignancies, including acute myeloid leukemia, diffuse large B-cell lymphoma, multiple myeloma, colorectal cancer, hepatocellular carcinoma and breast cancer. Anemia is common with anti-CD47 therapy because CD47 is ubiquitously expressed on senescent red blood cells (RBCs). However, in comparison to other antiCD7 mAbs, AK117 exhibits significantly weaker binding to human RBCs than tumor cells. In both in vitro and in vivo nonclinical studies, AK117 demonstrated robust anti-tumor activity without causing significant agglutination of human RBCs in vitro or anemia in monkeys.

Methods This is a first-in-human, Phase 1, multicenter, open label, single arm, dose escalation and dose expansion study of AK117 administered intravenously to adult subjects with relapsed/refractory advanced or metastatic solid tumors or lymphomas. The primary objective is to assess the safety and tolerability of AK117 monotherapy; and determine the maximum tolerated dose (MTD). Antitumor activity, PK, pharmacodynamic profile and immunogenicity of AK117 will be studied as secondary objectives. The dose escalation parts of the study uses a $3+3+3$ design to determine the Recommended Phase 2 Dose (RP2D) dose between $0.3 \mathrm{mg} / \mathrm{kg}$ to 45 $\mathrm{mg} / \mathrm{kg}$ QW. Dose escalation is performed exclusively in solid tumors; and any dose escalation cohort not exceeding the MTD may be expanded up to a maximum of approximately 18 subjects, with a minimum of 6 subjects with lymphomas, to provide additional clinical data to inform the optimal dose level and treatment schedule for tumor type-specific dose expansion and subsequent clinical studies. In the dose expansion phase, AK117 will be studied in cohorts of 30 subjects each with selected solid tumors and selected lymphomas.Subjects with active or prior autoimmune disease that may relapse, history of hemolytic anemia of any cause within 3 months of first dose, hemophagocytic lymphohistiocytosis, defects in RBC production; or defects in hemoglobin production or metabolism will not be eligible for this study.

Results N/A

Conclusions N/A

Trial Registration NCT04349969

Ethics Approval The study was approved by Bellberry Human Research Ethics Committee (Application No. 2020-02-016).

http://dx.doi.org/10.1136/jitc-2020-SITC2020.0384

\section{A FIRST-IN-HUMAN STUDY OF LEMZOPARLIMAB, A DIFFERENTIATED ANTI-CD47 ANTIBODY, IN SUBJECTS WITH RELAPSED/REFRACTORY MALIGNANCY: INITIAL MONOTHERAPY RESULTS}

${ }^{1}$ Jordan Berlin*, ${ }^{2}$ Wael Harb, ${ }^{3}$ Alex Adjei, ${ }^{4}$ Yan Xing, ${ }^{5}$ Paul Swiecicki, ${ }^{3}$ Mahesh Seetharam, ${ }^{6}$ Lakshminarayanan Nandagopal, ${ }^{7}$ Ajay Gopal, ${ }^{8} \mathrm{Cong} \mathrm{Xu}^{8}{ }^{8} \mathrm{Cong} \mathrm{Xu},{ }^{8}$ Yuan Meng, ${ }^{8}$ Linda Lee, ${ }^{8}$ Yonggang Zhao, ${ }^{8}$ Zhengyi Wang, ${ }^{8}$ Joan Huaqiong Shen. ${ }^{1}$ Vanderbilt University, Nashville, TN, USA; ${ }^{2}$ Horizon Oncology, Lafayette, IN, USA; ${ }^{3}$ Mayo Clinic, Rochester, MN, USA; ${ }^{4}$ City of Hope, Duarte, CA, USA; ${ }^{5}$ University of Michigan, Ann Arbor, MI, USA; ${ }^{6}$ University of Alabama, Birmingham, AL, USA; ${ }^{7}$ University of Washington, Seattle, WA, USA; ${ }^{8}$-Mab Biopharma, Gaithersburg, MD, USA

Background CD47 blockade using SIRP $\alpha-F c$ or anti-CD47 antibodies results in inhibition of the 'do not eat' signal and activation of phagocytosis and has emerged as a promising 